sexually active adolescent and young adult women is recommended, only in recent years has there been a major emphasis on repeat testing at 3 months after diagnosis to identify reinfection. ${ }^{8}$ Longer than recommended testing intervals could miss reinfections as these are likely to be of shorter duration than primary infections. What were the results of diagnostic tests done when pelvic inflammatory disease was diagnosed? Could secular changes in chlamydia test technologies or patient management have affected the size of risk identified in this study? Antigen detection methods used during 1995-2000 could have missed $50 \%$ of infections detectable by secondgeneration nucleic acid amplification tests available afterwards. ${ }^{9,10}$ Patients with pelvic inflammatory disease were once routinely admitted to hospital and diagnosis of ectopic pregnancy was determined surgically, but both transitioned predominantly to outpatient management. ${ }^{11}$ Because ascertainment of complications was based solely on hospital records, further work is needed to see if cohort members attended other primary care settings for milder presentations of complications.

Answers to these questions could help define causes of excess risk of reproductive complications seen after a positive chlamydia test and inform more effective interventions leading to improved outcomes. We look forward to further mining of this valuable dataset.
*Julius Schachter, Joan M Chow

Department of Laboratory Medicine (JS) and Department of Obstetrics, Gynecology, and Reproductive Sciences (JMC), University of California, San Francisco, CA 94143, USA julius.schachter@ucsf.edu

We declare no competing interests.

Copyright $\odot$ Schachter et al. Open Access article distributed under the terms of CC BY

1 Westrom L, Joesoef R, Reynolds G, et al. Pelvic inflammatory disease and fertility. Sex Transm Dis 1992; 19: 185-92.

2 Davies B, Turner KME, Frølund M, et al, and the Danish Chlamydia Study Group. Risk of reproductive complications following chlamydial testing: a population-based retrospective cohort study in Denmark. Lancet Infect Dis 2016; published online June 8. http://dx.doi.org/10.1016/S14733099(16)30092-5.

3 Bakken IJ, Ghaderi S. Incidence of pelvic inflammatory disease in a large cohort of women tested for Chlamydia trachomatis: a historical follow-up study. BMC Infect Dis 2009; 9: 130 .

4 Davies $\mathrm{B}$, Ward $\mathrm{H}$, Leung $\mathrm{S}$, et al. Heterogeneity in risk of pelvic inflammatory diseases after chlamydia infection: a population-based study in Manitoba, Canada. J Infect Dis 2014; 210 (suppl 2): S549-55.

5 Hillis SD, Owens LM, Marchbanks PA, Amsterdam LF, Mac Kenzie WR. Recurrent chlamydial infections increase the risks of hospitalization for ectopic pregnancy and pelvic inflammatory disease. Am J Obstet Gynecol 1997; 176 (1 pt 1): 103-07.

6 Lind I, Hoffmann S. Recorded gonorrhoea rates in Denmark, 1900-2010: the impact of clinical testing activity and laboratory diagnostic procedures. BMJOpen 2015; 5: e008013.

7 Haggerty CL, Totten PA, Tang G, et al. Identification of novel microbes associated with pelvic inflammatory disease and infertility. Sex Transm Infect 2016; published online Jan 29. DOI:10.1136/sextrans-2015-052285.

8 Workowski KA, Bolan GA. Sexually transmitted diseases treatment guidelines, 2015. MMWR Recomm Rep 2015; 64 (RR-03): 1-137.

9 Schachter J. Which test is best for chlamydia? Curr Opin Infect Dis 1999 12: 41-45.

10 Schachter J, Hook EW, Martin DH, et al. Confirming positive results of nucleic acid amplification tests (NAATs) for Chlamydia trachomatis: all NAATs are not created equal. J Clin Microbiol 2005; 43: 1372-73.

11 Scholes D, Satterwhite CL, Yu O, Fine D, Weinstock H, Berman S. Long-term trends in Chlamydia trachomatis infections and related outcomes in a U.S. managed care population. Sex Transm Dis 2012; 39: 81-88.

\title{
@ Equitable control of schistosomiasis and helminthiasis
}

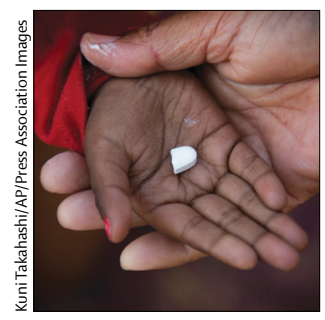

Published Online June 7, 2016 http://dx.doi.org/10.1016/ S1473-3099(16)30124-4 See Articles page 1065
Over the past decade, the continued high prevalence of some neglected tropical diseases (NTDs) has necessitated a revision of where and what kind of intensive control interventions are needed and what the associated targets are. ${ }^{1-4}$ Set within a global strategy of preventive chemotherapy, as endorsed by $\mathrm{WHO}$, the frontline public health method is routine co-administration by mass drug administration (MDA), of the anthelmintics praziquantel against schistosomiasis and albendazole against soiltransmitted helminthiasis., ${ }^{5.6}$ In 2001, an ambitious target was set within resolution 54.19 of the World Health Assembly to attain regular treatment coverage of at least $75 \%$ in all school-age children at risk of morbidity. With noted progress falling short of this target by 2010, in January, 2012, several substantial pledges and commitments were made at the London Declaration on NTDs with an additional World Health Assembly resolution 65.21, and both called for intensification of efforts to better rally resources and to ensure an adequate provision of medications.

In The Lancet Infectious Diseases, Nathan Lo and colleagues $^{7}$ show that striving towards $75 \%$ coverage should now be considered as the minimum standard of care in those communities targeted or deemed eligible for praziquantel and albendazole treatment. They describe novel cost-effectiveness analyses of current MDA strategies, based on infection prevalence thresholds to explore putative outcomes of alternative prevalence thresholds as summed over a period of 5 years, assuming $75 \%$ treatment coverage. Most 
interestingly, they show that, if $21 \cdot 3 \%$ of the population shifted to integrated treatment with praziquantel and albendazole, programme synergies would lead to a $40 \%$ reduction in implementation costs (as noted in the Article's appendix). Moreover, their analyses show annual preventive chemotherapy against schistosomiasis to be highly cost-effective in treatment of school-aged children at a prevalence of 5\% (95\% uncertainty interval [UI] 1.7-5.2; current guidelines state 50\%) and of the entire community at $15 \%(7 \cdot 3-18 \cdot 5)$. Annual MDA against soil-transmitted helminthiasis was highly costeffective in treatment of school-aged children at a prevalence of $20 \%$ (95\% UI 5.4-30.5\%; current guidelines state $20 \%$ ) and for the entire community at $60 \%$ (35.3-85.1).

Furthermore, Lo and colleagues ${ }^{7}$ show that earlier approaches have not improved equity of access to treatment. Nor would these lead to substantial disability-adjusted life-years averted. ${ }^{8}$ By investigation of the different scenarios, they surmise that much lower prevalence thresholds than WHO norms are more sensible. These could be used at international and national levels. ${ }^{8,9}$ However, whether these new norms for control efforts are entirely practical is not yet clear, because if their advice was closely followed, overestimation of the bottlenecks and strictures in logistics that need to be overcome in this new logical chain of events is likely. Without doubt, more information on best implementation strategies is needed to sensibly guide its transition and application such that expanded access is not counterproductive. Foremost, dramatic change is needed in our appraisal of these diseases by reducing existing barriers to expansion of treatment, irrespective of age, sex, and disability.

Using their analytical framework, Lo and colleagues ${ }^{7}$ estimate that treatment needs for Africa are six times higher than current guidelines for praziquantel and two times higher for albendazole. Thus, the consequences for country programming are immense; the MDA strategies might prove to be cost-effective, but the involved total budgets and other investments needed will be substantial in absolute terms for each country involved. Implementation of the suggested framework is a major undertaking, in view of the paucity of resources in other priority areas within NTD programmes and other health domains. From a budgetary perspective, a realistic limited budget might actually facilitate up-scaled, stepwise implementation and prevent rejection as unrealistic in real life. In their analyses, Lo and colleagues used county-specific affordability standards related to their available national income. In all scenarios, ${ }^{1,10}$ increased drug subsidies ${ }^{11-13}$ and donations from pharmaceutical companies, strong political will, increased logistical support, and improved epidemiological surveillance to monitor for drug resistance are needed. At lower prevalence levels, the at-risk vulnerable populations ${ }^{14}$ may be living in poverty and be harder to reach ${ }^{11}$ and lower compliance will lead to lower returns. Additionally, Lo and colleagues note the extra effort and resources needed nationally to improve the quality of the existing expanded MDA programmes.

Although Lo and colleagues ${ }^{7}$ reason that treatment expansion is both financially and geographically appealing, extension of coverage is also soundly justified ethically. ${ }^{11}$ A key feature will be securing additional pledges from the pharmaceutical sector to provide stocks to ensure continuous drug delivery pipelines. Similarly, we need to put in place strategies to ensure that treatment fatigue among those receiving MDA treatment over many years within the community does not dampen the recipients' compliance and that their demand for treatment continues to match the available and future supply through national services and donors. In revising preventive chemotherapy guidelines, broader quantitative and qualitative evidence should also be incorporated and collected to optimise the implementation of treatment campaigns, which often have low uptake. Although these interventions are potentially cost-effective, they might not be all affordable within countries' tight budgets. At least, they are a next, essential step in the promotion of universal access for the prevention and treatment of NTDs as also envisaged in WHO's Expended Project for the Elimination of NTDs (ESPEN) and the Sustainable Development Goals.

\section{*Louis Niessen, Russell Stothard \\ Department of Clinical Sciences, Liverpool School of Tropical Medicine, Liverpool L3 5QA, UK louis.niessen@lstmed.ac.uk}

$\mathrm{LN}$ and RS participate in the four-country research programme consortium COUNTDOWN, funded by the Research and Evidence Division of the Department for International Development. 
1 Hotez PJ, Molyneux DH, Fenwick A, Ottesen E, Sachs SE, Sachs JD. Incorporating a rapid-impact package for neglected tropical diseases with programs for HIV/AIDS, tuberculosis and malaria. PLoS Med 2006; 3: $576-84$.

2 WHO. A generic framework for the control, elimination, and eradication of neglected diseases. 2016. http://apps.who.int/iris/bitstream/10665/20 5080/1/WHO_HTM_NTD_2016.6_eng.pdf (accessed June 5, 2016).

3 Webster JP, Molyneux DH, Hotez PJ, Fenwick A. The contribution of mass drug administration to global health: past, present and future. Philos Trans R Soc Lond B Biol Sci 2014; 369: 20130434

4 Yamey G, Torreele E. The world's most neglected diseases. BMJ 2002; 325: 176-77.

5 Stothard JR, Bustinduy A, Montresor A. Preventive chemotherapy for schistosomiasis and soil-transmitted helminthiasis by cotreatment with praziquantel and albendazole. Clin Investig (Lond) 2014; 4: 163-76.

6 Stothard JR, Sousa-Figueiredoabab JC, Navaratnam A. Advocacy, policies and practicalities of preventive chemotherapy campaigns for African children with schistosomiasis. Expert Rev Anti Infect Ther 2013; 1: 733-52.

7 Lo NC, Lai Y-S, Karagiannis-Voules D-A, et al. Assessment of global guidelines for preventive chemotherapy against schistosomiasis and soil-transmitted helminthiasis: a cost-effectiveness modelling study. Lancet Infect Dis 2016; published online June 7. http://dx.doi.org/10.1016/ S1473-3099(16)30073-1.
8 de Vlas SJ, Stolk WA, le Rutte EA, et al. Concerted efforts to control or eliminate neglected tropical diseases: how much health will be gained? PLoS Negl Trop Dis 2016; 10: e0004386.

9 Hollingsworth TD, Adams ER, Anderson RM, et al. Quantitative analyses and modelling to support achievement of the 2020 goals for nine neglected tropical diseases. Parasit Vectors 2015; 8: 630.

10 WHO. WHO guideline on country pharmaceutical pricing policies. Geneva: World Health Organization, 2013.

11 WHO. Health systems financing: the path to universal coverage. Geneva: World Health Organization, 2010.

12 Niessen LW, Khan JA. Universal access to medicines. Lancet 2016; 387: 9-11.

13 Cameron A, Ewen M, Ross-Degnan D, Ball D, Laing R. Medicine prices, availability, and affordability in 36 developing and middle-income countries: a secondary analysis. Lancet 2009; 373: 240-49.

14 van Doorslaer E, O'Donnell O, Rannan-Eliya RP. Effect of payments for health care on poverty estimates in 11 countries in Asia: an analysis of household survey data. Lancet 2006; 368: 1357-64.

\section{@ Global maternal group B streptococcus colonisation}

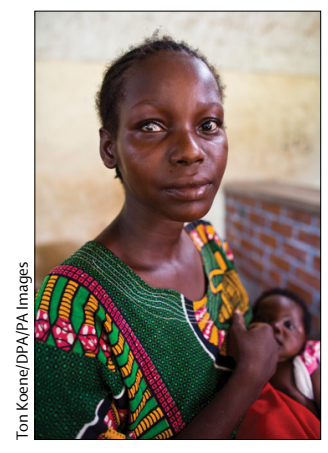

Published Online May 25, 2016 http://dx.doi.org/10.1016/ S1473-3099(16)30072-X See Articles page 1076
Globally, neonatal mortality remains unacceptably high, with little change in the death rate since 1990, despite a halving of under-5 mortality in the same timeframe. ${ }^{1}$ Group B streptococcus is the leading cause of neonatal mortality in the $\mathrm{UK}^{2}$ and the $\mathrm{USA}^{3}$ with long-term adverse neurodevelopmental outcomes in up to $50 \%$ of survivors. ${ }^{4}$ In low-income and middle-income countries, little is known about the role of group B streptococcus in neonatal deaths. ${ }^{5}$ Disease estimates in these countries might be an underestimation of the true disease burden because the need for selective growth media to identify group B streptococcus has been under-recognised. ${ }^{5,6}$

Group B streptococcus acquisition occurs through vertical transmission in $15-50 \%$ of infants born to a group B streptococcus colonised mother. ${ }^{7}$ Maternal colonisation is a prerequisite for early-onset and a risk factor for late-onset disease. ${ }^{3}$ Intrapartum antibiotics can prevent early-onset group B streptococcal disease, and, as a consequence, in many high-income countries this form of the disease has become much less common. ${ }^{3}$ Apart from the dangers of widespread antibiotic use, intrapartum antibiotics pose logistical and financial problems in low-income and middle-income settings and are poorly implemented. ${ }^{8,9}$ An effective and costefficient alternative to intrapartum antibiotics is to implement a maternal vaccination strategy. However, because estimates of disease burden and disease-causing serotypes are only available in a few countries, the development of a global vaccine has proven problematic.

To estimate the true burden of group B streptococcal disease, measurement of maternal colonisation by country might be useful, because maternal colonisation is the main risk factor for early-onset group B streptococcal disease. In a previous systematic review, Stoll and colleagues $^{10}$ showed that, by contrast with group B streptococcus invasive disease prevalence, maternal group B streptococcus colonisation rates in low-income and middle-income settings were similar to those of high-income countries. However, laboratory methods precluded extrapolation of the data to provide a global estimate. Therefore, if differences in disease rates are solely a result of a combination of intrapartum antibiotics and laboratory methods, then using standardised laboratory methods should show a variation in maternal colonisation in line with disease estimates.

In The Lancet Infectious Diseases, Gaurav Kwatra and colleagues ${ }^{11}$ identified prospective studies that used standardised laboratory methods for group B streptococcus identification to calculate a robust estimate of the true burden of maternal colonisation. Their findings from 73791 pregnant women in 78 studies across 37 countries showed an overall global estimate of $17.9 \%(95 \% \mathrm{Cl} 16 \cdot 2-19 \cdot 7)$. Their results highlight substantial variation between and within countries, with the prevalence of colonisation lowest 